\title{
Assessment of validity evidence for the RobotiX robot assisted surgery simulator on advanced suturing tasks
}

\author{
Erik Leijte ${ }^{1,2^{*}}$, Ivo de Blaauw², Camiel Rosman ${ }^{1}$ and Sanne M. B. I. Botden²
}

\begin{abstract}
Background: Robot assisted surgery has expanded considerably in the past years. Compared to conventional open or laparoscopic surgery, virtual reality (VR) training is an essential component in learning robot assisted surgery. However, for tasks to be implemented in a curriculum, the levels of validity should be studied for proficiency-based training. Therefore, this study was aimed to assess the validity evidence of advanced suturing tasks on a robot assisted VR simulator.

Method: Participants were voluntary recruited and divided in the robotic experienced, laparoscopic experienced or novice group, based on self-reported surgical experience. Subsequently, a questionnaire on a five-point Likert scale was completed to assess the content validity. Three component tasks of complex suturing were performed on the RobotiX simulator (Task1: tilted plane needle transfer, Task: 2 intracorporal suturing, Task 3: anastomosis needle transfer). Accordingly, the outcome of the parameters was used to assess construct validity between robotic experienced and novice participants. Composite scores (0-100) were calculated from the construct parameters and corresponding pass/fail scores with false positive (FP) and false negative (FN) percentages.
\end{abstract}

Results: Fifteen robotic experienced, 26 laparoscopic experienced and 29 novices were recruited. Overall content validity outcomes were scored positively on the realism (mean 3.7), didactic value (mean 4.0) and usability (mean 4.2). Robotic experienced participants significantly outperformed novices and laparoscopic experienced participants on multiple parameters on all three tasks of complex suturing. Parameters showing construct validity mainly consisted of movement parameters, needle precision and task completion time. Calculated composite pass/fail scores between robotic experienced and novice participants resulted for Task 1 in 73/100 (FP 21\%, FN 5\%), Task 2 in 85/100 (FP 28\%, FN 4\%) and Task 3 in 64/100 (FP 49\%, FN 22\%).

Conclusion: This study assessed the validity evidence on multiple levels of the three studied tasks. The participants score the RobotiX good on the content validity level. The composite pass/fail scores of Tasks 1 and 2 allow for proficiency-based training and could be implemented in a robot assisted surgery training curriculum.

Keywords: Robotic surgery, Virtual reality simulation, Proficiency based training, Validation

\footnotetext{
* Correspondence: erik.leijte@radboudumc.nl

'Department of Surgery, Radboud University Medical Center, Geert grooteplein 10 route $618,6500 \mathrm{HB}$ Nijmegen, The Netherlands ${ }^{2}$ Department of Pediatric Surgery, Radboud University Medical Center, Geert grooteplein 10 route $618,6500 \mathrm{HB}$ Nijmegen, The Netherlands
}

(c) The Author(s). 2020 Open Access This article is licensed under a Creative Commons Attribution 4.0 International License, which permits use, sharing, adaptation, distribution and reproduction in any medium or format, as long as you give appropriate credit to the original author(s) and the source, provide a link to the Creative Commons licence, and indicate if changes were made. The images or other third party material in this article are included in the article's Creative Commons licence, unless indicated otherwise in a credit line to the material. If material is not included in the article's Creative Commons licence and your intended use is not permitted by statutory regulation or exceeds the permitted use, you will need to obtain permission directly from the copyright holder. To view a copy of this licence, visit http://creativecommons.org/licenses/by/4.0/. The Creative Commons Public Domain Dedication waiver (http://creativecommons.org/publicdomain/zero/1.0/) applies to the data made available in this article, unless otherwise stated in a credit line to the data. 


\section{Background}

Robot assisted surgery has been widely accepted during the past years and continues to grow which leads to more surgeons being trained in robot assisted surgery [1]. Training of robot assisted surgery is often compared to the training of an airline pilot, because both deal with complex technology and have very limited room for errors, which could result in severe complications. Therefore, these circumstances demand an extensive standardized training curriculum before a surgical trainee is fit to 'fly' [2].

There are multiple modalities used to learn robot assisted surgery [2, 3]. Proctoring often consists of an external expert providing direct supervision during surgery. Although this method has never proven its efficacy it is generally accepted that it allows for a safe and interactive learning. However, proctoring is expensive when required for a more extensive period [4]. Mentoring using a mentor console provides a safe collaboration between the trainee and a local experienced mentor [5]. Unfortunately, this method of training is limited in availability of an additional 'mentor' console and requires additional informed consent. Before proctoring or mentoring, simulation models are used to practice robotic skills or procedures in a safe environment. Simulation models primarily consist of virtual reality (VR) simulation, inanimate models and live animal or cadaveric training. While cadaveric training has the benefits of the realistic anatomy and the opportunity for procedural training, it remains costly and comes with ethical concerns $[6,7]$. The training with inanimate models such as $3 \mathrm{D}$-printed anatomical structures is a safe and realistic training method, but limited due to the requirement of training instruments and access to a live console. Therefore, the use of VR simulation is a widely accepted effective method to train robot assisted surgery from basic and advanced skills to procedural training [8]. The training with VR simulators is already a proven valuable tool for laparoscopic surgery and as a possible preoperative warm up [8-10].

However, for the use of VR simulation validation studies are required to determine the usefulness of training [11-13]. This allows for training aimed at improving proficiency [14]. Most validation studies on surgical robot simulators performed are aimed at basic surgical tasks and are assessed between novice and robot experienced participants [14]. Then again, the main advantage of robot assisted surgery is expected during complex tasks, which often require suturing skills and working in a small space. Therefore, laparoscopic participants are also a target group for learning robot assisted surgery besides novices [15]. The goal of this study is to collect the validity evidence according to Messick's contemporary framework for advanced robot assisted tasks on the
RobotiX robot assisted VR simulator and to establish a proficiency score $[11,16]$. Besides, novice participants, we also take in account the performance of laparoscopic experienced participants.

\section{Methods \\ Participants}

Participants included in this study were voluntary recruited at the Radboud university medical center Nijmegen, the Netherlands and during the European Association of Urology congress Copenhagen 2018. To prevent influence of work fatigue, the simulations were conducted outside of the OR and only during the morning or afternoon. The subjects were dived in either of the three groups based on their self-reported surgical experience. Participants with a medical background and understanding of minimal invasive surgery but without clinical surgical experience were selected in the 'novice group' as a control group. Participants with clinical laparoscopic experience and without robot assisted experience were allotted to the 'laparoscopic experience group'. The laparoscopic experienced group was included in this study because they are unexperienced with robot assisted surgery and most likely the next participants to learn robot assisted surgery and therefore, the target group. Finally, the robot assisted experienced participants with $>10$ robot assisted clinical procedures experience were allotted to the 'robotic experience group'.

\section{Questionnaire}

For the content validity evidence, a previously used questionnaire was adapted for this study [17-19]. The questionnaire consisted of a section regarding the participants informed consent, demographic information and surgical experience and can be found in Supplemental 1. The second section was completed after performing the three tasks and consisted of multiple questions on a fivepoint Likert scale. These questions were divided in the domains; realism, didactic value and usability per task. With ' 1 ' representing 'in strong disagreement', ' 3 ' as a 'neutral opinion' and ' 5 ' being in 'strong agreement' [20]. Outcomes of $>3.5$ were considered as positive scores. The realism was assessed using questions concerning the perceived realism, grasper manipulation, tissue handling and on-screen response. The didactic value contained questions regarding the value to train inexperienced and experienced surgeons, and the value to assess the skills of a trainee. The usability was scored by the participants on the user-friendliness of the simulator interface and the appeal of the system to train for this task. 


\section{Simulator and metrics}

The standard supplied setup of the RobotiX Mentor (3D systems, Colorado, USA) platform was used in this study (Fig. 1). The system consisted of an operating tower containing the computer with screen and the console unit which functions as the workspace with the simulator master controls and the 3D viewer. The platform installation and user instruction were provided by 3D Systems to ensure correct use. The system is designed to mimic the da Vinci ${ }^{\circ}$ Surgical System (Intuitive Surgical Inc., California, USA). This is done by the freehand controls, $3 \mathrm{D}$ view and similar ergonomic workspace setting which can be personally adjusted. The supplied software 'Mentorlearn' was used for tracking the performance parameters per participant. The software kept track of twelve to twenty-five parameters per tasks from which the most clinically significant ones were selected by experienced surgeons to be included in this study. The included parameters were accordingly divided in three domains consisting of; movement, safety and task specific parameters. The parameter definitions are stated in Table 1.

\section{Tasks}

The tasks selected for this study were based on their representation of skills required during complex suturing surgery. This is where the most advantage of the robotic assistance is to be expected compared to conventional minimally invasive surgery.

Task 1: Railroad track (Fig. 2) is a needle transfer task in a tilted plane without knot tying. The supplied needle and thread had to be transferred through multiple dots in a matrass pattern. To complete the task the needle had to be anchored in the virtual ball.

Task 2: Intracorporal suturing (Fig. 3) is a standard suturing task where two surgical knots had to be placed on a virtual suturing pad. The system gave instructions

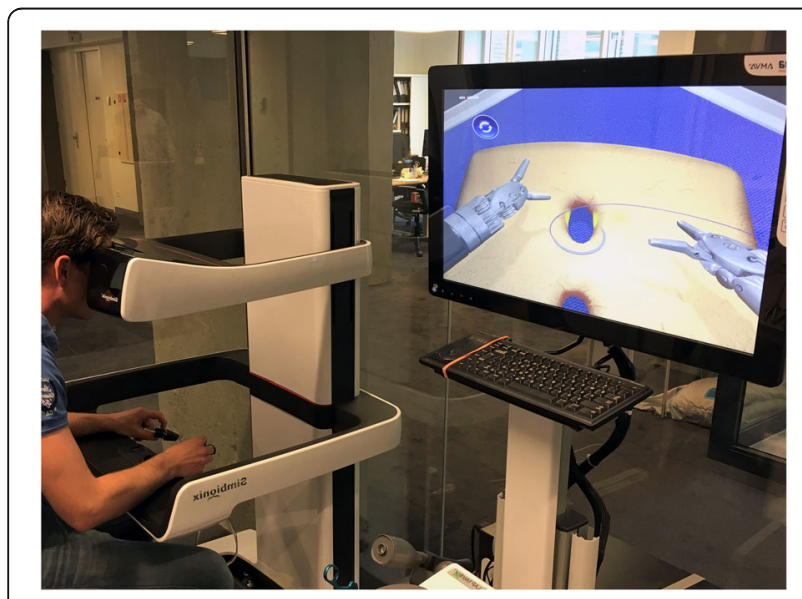

Fig. 1 setup of the RobotiX Mentor VR simulator as used in this study during the tasks which was finished when two knots have been placed.

Task 3: Vaginal cuff closure (Fig. 4) simulates an anastomosis needle transfer task without knot tying. The task was performed with a barbed wire suture which is used to close a vaginal cuff (after hysterectomy) with guidance from highlighted dots. Once the required transfers were made and the suture was cut, the task was completed.

\section{Protocol}

Upon entering, the study participants completed the first part of the questionnaire regarding their demographics and surgical experience. The 'response validity' was maintained by having a single researcher giving the handling, system instructions and explaining the written 'Mentorlearn' task. To attain familiarity with the system, participants first performed two basic tasks concerning the wristed capability and tissue handling. Subsequently the three selected suturing tasks were performed. A maximum of 20 min was given for the completion of the tasks and performance outcomes were saved by the 'Mentorlearn' software. At completion of the performed tasks the participant completed the remainder of the questionnaire on the realism, didactic value and usability per task, to assess the 'content source of validity'. This assessment is mainly based on the opinion of the robotic experienced participants, because they have the clinical experience. However, the novices and laparoscopic experienced participants are the possible future trainees for robot assisted surgery and were, therefore, included. The performance scores of each group were used to assess the 'relation to other variables validity' by comparing performance outcomes for parameters being statistically significantly different and thus showing construct validity. Accordingly, a composite score was calculated with the construct parameters to determine a pass/fail score for the 'consequence of the test validity'.

\section{Statistical analysis}

Data analysis was performed using the Statistical package for social sciences (SPSS) version 25 (IBM Corp., New York, USA). All $P$-values $<0.05$ were considered statistically significant.

The content and relation to other variables validity were analyzed with the outcomes from the questionnaire and the performance scores using independent $t$-test between each group after testing for normal distribution. Statistically significant different performance outcomes between novice and robotic experienced participants were included for the composite score calculation. Parameters resulting in 'better' performance for the novice group were excluded for the composite score calculation. The composite score was calculated from 
Table 1 parameter definitions of the selected clinically relevant parameters

\begin{tabular}{ll}
\hline Movement parameters & Definition \\
\hline Path length (left/right) & Distance travelled by instrument in millimeter \\
Movements (left/right) & Number of movements made by instrument \\
Entrance and exit points & Total number of entrance and exit points \\
Distance scope and tissue & Average distance between scope and vaginal cuff in millimeter \\
Safety parameters & \\
$\begin{array}{l}\text { Inaccurate punctures } \\
\text { Suture breakage }\end{array}$ & Number of needle punctures not within relevant target mark \\
Instrument collisions & Number of times the suture broke due to excessive force \\
Times out of view & Number of times instruments collided \\
Dropped needles & Number of times the users instrument was held out of view \\
Unnecessary piercing & Number of times a needle was dropped \\
Task specific parameters & Number of unnecessary piercing points \\
Total time & \\
Total errors & Total time in seconds \\
Needle precision & Total number of errors made \\
Needle passages & Percentage of needle punctures which are on relevant target mark \\
Precise needle passages & Total number of needle passages made \\
Total knots & Number of needle passages on relevant mark \\
Surgeon knots & Total number of knots made \\
\hline
\end{tabular}

the mean value of the selected parameters after linear normalization ranging from 0 to 100 with the latter being the highest score.

Consequence validity was analyzed by using the calculated composite score for a pass/fail cutoff value, which was determined by the contrasting groups method. For this method the model by Jorgensson et al. was used and adapted to incorporate the mean score and standard deviation of three groups to calculate the optimal pass/fail scores [21]. Additionally, this model calculates the theoretical false positive and false negatives, which can be used as an addition to the absolute false positives and false negatives,

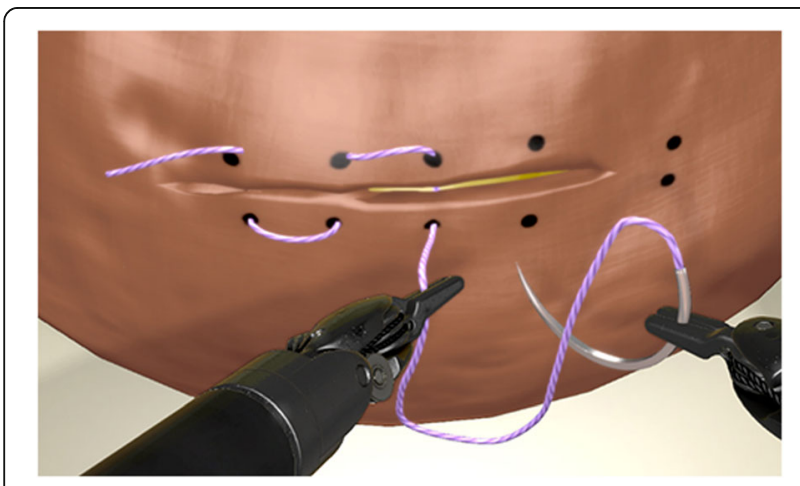

Fig. 2 Screenshot of Task 1 Railroad track (tilted plane needle transfer), figure provided by 3D systems because these are prone to be unreliable for small sample sizes and outliers [21].

\section{Results}

\section{Demographics}

A total of 70 participants were included of which 29 novices, 26 laparoscopic experienced and 15 robotic experienced participants with the characteristics shown in Table 2. The novice group consisted of medical students with a mean age of 24 years without any laparoscopic or robot assisted experience. The laparoscopic group contained seventeen residents in training and nine

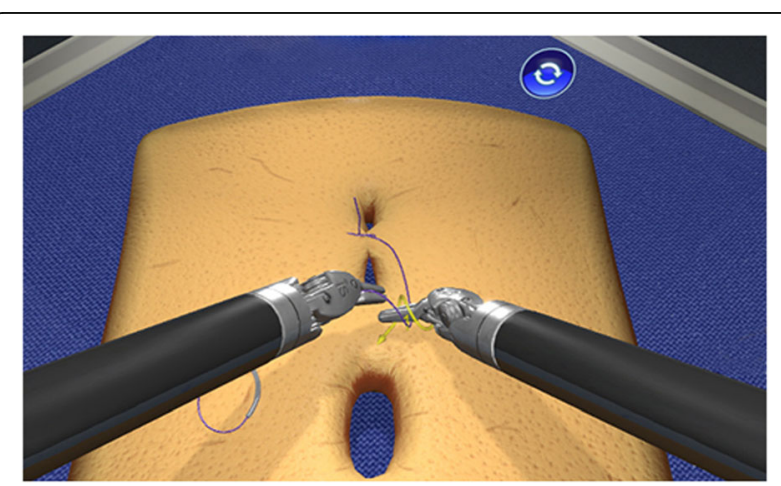

Fig. 3 Screenshot of Task 2 Intracorporal suturing, figure provided by 3D systems 


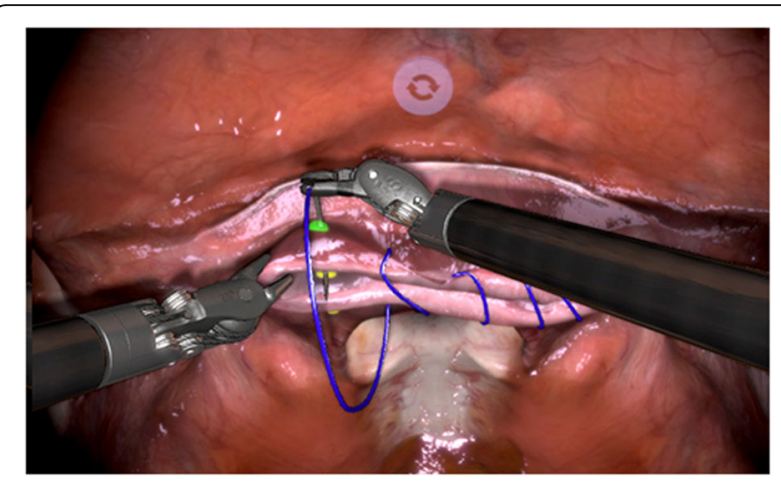

Fig. 4 Screenshot of Task 3 Vaginal cuff closure (anastomosis needle transfer), figure provided by 3D systems

specialized surgeons from surgical, urologic and gynecologic specialties. The mean age was 35 years and $92 \%$ right-handed dexterity. The robotic experienced group consisted of five residents in their fourth till sixth year of specialty training and ten specialized surgeons. Robotic experience ranged from 0 to $>50$ basic procedures completed by seven participants and $>50$ basic procedures completed by eight participants. The number of advanced procedures ranged from 0 to 50 for eight participants with the remaining seven participants having completed $>50$ advanced procedures. Mean age and dexterity in the robotic experienced group was 43 years and $73 \%$ right-handedness respectively.

\section{Content (realism, didactic value and usability)}

The opinion values of the three tasks are shown in Table 3. The overall score for the realism, didactic value and usability was rated positively. The robotic experienced participants scored the usability of the system significantly lower than the novice group for all tasks ( $p$-values 0.007, 0.002 and 0.048 respectively). However, the lowest mean usability score by the robotic experienced participants was 3.9, which is still rated good. The realism was scored lowest by the robotic experienced participants on all tasks (3.5, 3.4 and 3.5 respectively) resulting in a neutral to moderate positive opinion on the realism. The lowest realism scores from the robotic experienced participants were found at the behavior of sutures running through the tissue of Task 1 and 3 (mean 3.3 and 3.1) and the thread behavior at Task 2 (mean 3.1). The highest mean realism scores from the robotic experienced were found for the realism to mimic needle transfer at Task 1 (mean 3.7) and the realistic onscreen response during Task 2 and 3 (both mean 3.9). The laparoscopic participants scored the realism of Task 3 statistically significantly higher than the robotic experienced (4.1 versus $3.5, p=0.009$ ) and novice group (4.1 versus 3.6, $p=0.005$ ). This is also seen at the realism sub questions 'realism to mimic vaginal cuff closure' (laparoscopic 4.2 versus robot 3.5 and novices 3.6, $p=0.018$ and $p=0.024$ ) and suture behavior (laparoscopic 3.9 versus robot 3.1 and novices $3.4, p=0.006$ and $p=0.016$ ). This indicates a disagreement in realism perception between the laparoscopic participants and the remaining groups. All three groups agreed concerning the didactic value, scoring it positively for all tasks (overall means of 3.9, 4.1 and 4.0 respectively). The specific lowest didactic value scores by the robotic experienced participants were found for the didactic value to train experienced surgeons on all tasks (mean 3.8, 3.7 and 3.6, respectively).

\section{Relation with other variables (construct) \\ Task 1}

The mean performance score of Task 1 are presented in Table 4. Statistically significant differences in performance outcomes between the robotic experienced versus novices and laparoscopic group was shown for all the included movement parameters, as well as the 'inaccurate punctures', 'instrument collisions', 'needle precision' and 'total time' parameters ( $p$-values $<0.001-0.014)$. The laparoscopic experienced participants only scored significantly better than the novice participants for the 'total time' parameter ( $475 \mathrm{~s}$ versus $597 \mathrm{~s}, p=0.047$ respectively).

\section{Task 2}

In the second task the robotic experienced group performed statistically significantly higher than the novice group on the parameters; 'entrance and exit points', 'dropped needles', 'unnecessary needle piercing', 'suture breakage', 'needle precision' and 'total time' as shown in Table 5. Similar results were found when comparing the robotic with the laparoscopic experienced group regarding 'unnecessary needle piercing', 'suture breakage', 'needle precision' and 'total time' parameters. Although, there was a difference in the parameter 'needle out of view' in favor of the robotic experienced participants, this was not statistically significantly different. The 'total knots' and 'surgeon knots' were not significantly different between the groups, although, the system knot scoring was strict and did not allow for knot variations. The laparoscopic experienced participants significantly outperformed the novice group on the 'dropped needles', 'unnecessary piercing points' and 'total time' parameters ( $p$-values $0.039,0.036$ and $<0.001$ respectively). At Task 2 a technical error occurred resulting in loss of performance data of one novice and one robotic experienced participant. 
Table 2 Demographics

\begin{tabular}{|c|c|c|c|}
\hline & Robotic experience & Laparoscopic experience & Novices \\
\hline $\mathrm{N}$ & 15 & 26 & 29 \\
\hline Mean age (SD) & $43(10.8)$ & $35(5.5)$ & $24(2.4)$ \\
\hline Sex (male/female) & $14 / 1$ & $15 / 11$ & $12 / 17$ \\
\hline Dexterity (right/left/ambidextrous) & $11 / 0 / 3$ & $24 / 2 / 0$ & $25 / 4 / 0$ \\
\hline \multicolumn{4}{|l|}{ Surgical skill level } \\
\hline Medical student & - & - & 29 \\
\hline \multicolumn{4}{|l|}{ Resident in training } \\
\hline $1-2$ years & 0 & 2 & - \\
\hline $3-4$ years & 1 & 11 & - \\
\hline $5-6$ years & 4 & 4 & - \\
\hline Surgeon & 10 & 9 & - \\
\hline \multicolumn{4}{|l|}{ Specialty } \\
\hline None & - & - & 29 \\
\hline Surgery & 3 & 8 & - \\
\hline Urology & 11 & 4 & - \\
\hline Gynecology & 1 & 10 & - \\
\hline Pediatric & 0 & 4 & - \\
\hline \multicolumn{4}{|c|}{ Overall laparoscopic experience (years) } \\
\hline 0 & 1 & 0 & 29 \\
\hline$<1$ & 0 & 1 & - \\
\hline $1-5$ & 5 & 18 & - \\
\hline $5-10$ & 1 & 2 & - \\
\hline$>10$ & 8 & 5 & - \\
\hline \multicolumn{4}{|l|}{ Robot assisted experience } \\
\hline \multicolumn{4}{|l|}{ Basic procedures } \\
\hline 0 & 1 & 25 & 29 \\
\hline$<10$ & 2 & 1 & - \\
\hline $11-30$ & 4 & 0 & - \\
\hline $31-50$ & 0 & 0 & - \\
\hline$>50$ & 8 & 0 & - \\
\hline \multicolumn{4}{|l|}{ Advanced procedures } \\
\hline 0 & 2 & 26 & 29 \\
\hline $1-20$ & 6 & - & - \\
\hline $21-50$ & 0 & - & - \\
\hline$>50$ & 7 & - & - \\
\hline
\end{tabular}

$S D$ standard deviation. Procedures with intracorporal suturing were considered advanced

\section{Task 3}

Table 6 shows the mean performance outcomes for Task 3. Statistically significant better performance scores of the robotic experienced compared to the novice group were found for the following four parameters; 'path length left', 'instruments collisions', 'precise needle passages' and 'total time' ( $p$-values $0.015,0.001,0.032$ and 0.024 respectively). Interestingly, the laparoscopic experienced group was significantly outperformed by the robotic group on six parameters; 'path length left' $(p<$ $0.001)$, 'movements left' $(p=0.018)$, 'entrance and exit points' $(p=0.013)$, 'instruments collisions' $(p=0.002)$, 'needle passages' $(p=0.013)$ and 'total time' $(p=0.040)$. Although, some statistical differences were found in the previous tasks for laparoscopic experienced versus novice participants, none were apparent in this task. The robotic experienced participants had their instruments more often out of view than the novice participants (32 
Table 3 Mean opinion scores (standard deviation) for the three domains of the questionnaire

\begin{tabular}{|c|c|c|c|c|c|c|c|}
\hline & Robotic experience & Laparoscopic experience & Novices & Total group & $\boldsymbol{p}$-values & & \\
\hline Task 1 & & & & & R vs N & L vs N & $R$ vs $L$ \\
\hline Realism & $3.5(0.7)$ & $3.5(0.6)$ & $3.7(0.5)$ & $3.6(0.6)$ & 0.230 & 0.106 & 0.853 \\
\hline Didactic value & $4.0(0.9)$ & $3.7(1.0)$ & $4.0(0.5)$ & $3.9(0.8)$ & 0.892 & 0.156 & 0.354 \\
\hline Usability & $3.9(0.6)$ & $4.2(0.6)$ & $4.4(0.5)$ & $4.1(0.6)$ & 0.007 & 0.195 & 0.161 \\
\hline \multicolumn{8}{|l|}{ Task 2} \\
\hline Realism & $3.4(0.8)$ & $3.8(0.5)$ & $3.8(0.5)$ & $3.7(0.6)$ & 0.078 & 0.975 & 0.082 \\
\hline Didactic value & $4.0(0.7)$ & $4.2(0.5)$ & $4.2(0.4)$ & $4.1(0.6)$ & 0.274 & 0.923 & 0.360 \\
\hline Usability & $3.9(0.6)$ & $4.3(0.6)$ & $4.5(0.5)$ & $4.2(0.6)$ & 0.002 & 0.131 & 0.094 \\
\hline \multicolumn{8}{|l|}{ Task 3} \\
\hline Realism & $3.5(0.7)$ & $4.1(0.5)$ & $3.6(0.4)$ & $3.7(0.6)$ & 0.470 & 0.005 & 0.009 \\
\hline Didactic value & $3.9(0.8)$ & $4.1(0.8)$ & $4.1(0.6)$ & $4.0(0.7)$ & 0.386 & 0.801 & 0.564 \\
\hline Usability & $4.0(0.7)$ & $4.2(0.6)$ & $4.4(0.5)$ & $4.2(0.6)$ & 0.048 & 0.227 & 0.335 \\
\hline
\end{tabular}

Data in this table represents mean scores and standard deviation. Statistical differences were calculated using independent t-test. A $p$-value of $<0.05$ was considered statistically significant (displayed in bold)

versus 16 times, $p=0.030$ ). Therefore, the 'times out of view' parameter could not be included in the composite score. Additionally, the robotic experienced group worked significantly closer on the target tissue than the novice and laparoscopic groups as is seen in the 'distance scope and tissue' parameter $(94 \mathrm{~mm}$ versus 120 $\mathrm{mm}$ and $116 \mathrm{~mm} . p<0.001$ and $p=0.001$ respectively). There were less unnecessary needle piercings in the robotic experienced group compared to both other groups (mean 10.8 versus 14.4 and 13.1), However, this was not statistically significant.

\section{Sub-expert analysis}

In order to determine the influence of higher robotic assisted surgical experience, a sub group of robotic experienced participants with $>50$ advanced procedures $(n=7)$ was used. This sub-expert group resulted in construct validity for the same parameters as the robotic experience group compared to the novice group and was therefore not used in further analysis.

\section{Consequences (composite score and contrasting group)}

Calculation of the composite score per task led to a composite score for Task 1 consisting of the parameters; 'path length left', 'path length right', 'movements left', 'movements right', 'inaccurate punctures', 'instrument collisions', 'needle precision' and 'total time' parameters. The composite score of Task 2 was calculated with the parameters; 'suture breakage', 'entrance and exit points', 'dropped needles', 'unnecessary needle piercing', and

Table 4 Mean (SD) performance outcomes per group on Task 1

\begin{tabular}{|c|c|c|c|c|c|c|}
\hline Task 1: Railroad track & Robotic experience & Laparoscopic experience & Novices & $\boldsymbol{P}$-values & & \\
\hline Movements & $N=15$ & $N=26$ & $N=29$ & R vs N & L vs N & R vs $L$ \\
\hline Path length left & 1655 (478) & $3469(1577)$ & $4157(1870)$ & $<0.001$ & 0.148 & $<0.001$ \\
\hline Path length right & $2196(771)$ & $3879(2144)$ & $3885(1720)$ & 0.001 & 0.991 & 0.001 \\
\hline Movements left & $168(57)$ & $342(159)$ & $422(176)$ & $<0.001$ & 0.085 & $<0.001$ \\
\hline Movements right & $234(76)$ & $394(191)$ & $425(170)$ & $<0.001$ & 0.533 & 0.001 \\
\hline \multicolumn{7}{|l|}{ Safety } \\
\hline Inaccurate punctures & $5.5(3.1)$ & $10.9(7.3)$ & $13.4(9.4)$ & $<0.001$ & 0.274 & 0.002 \\
\hline Instrument collisions & $4.0(3.9)$ & $13.9(13.9)$ & $15.9(10.5)$ & $<0.001$ & 0.544 & 0.002 \\
\hline Times out of view & $1.3(1.0)$ & $2.4(4.0)$ & $1.7(2.1)$ & 0.663 & 0.435 & 0.581 \\
\hline \multicolumn{7}{|l|}{ Task specific } \\
\hline Needle precision & $63(15)$ & $48(20)$ & $47(19)$ & 0.007 & 0.926 & 0.014 \\
\hline Total errors & $13.9(12.1)$ & $11.1(5.5)$ & $11.3(3.3)$ & 0.452 & 0.896 & 0.322 \\
\hline Total time & $265(92)$ & $475(219)$ & $597(225)$ & $<0.001$ & 0.047 & $<0.001$ \\
\hline
\end{tabular}

Data in this table represents mean performance scores and standard deviation. $R$ Robotic experienced, $L$ Laparoscopic experienced, $N$ Novices. $P$-values were calculated using independent $\mathrm{t}$-test, values of $<0.05$ were considered statistically significant (displayed in bold). 
Table 5 Mean (SD) performance outcomes per group on Task 2

\begin{tabular}{|c|c|c|c|c|c|c|}
\hline Task 2: intracorporal suturing & Robotic experience & Laparoscopic experience & Novices & $\boldsymbol{P}$-values & & \\
\hline Movements & $n=14$ & $n=26$ & $n=28$ & R vs $N$ & L Vs N & $\overline{R \text { vs } L}$ \\
\hline Entrance and exits points & $5.7(2.1)$ & $7.7(4.6)$ & $11.4(8.8)$ & 0.003 & 0.064 & 0.075 \\
\hline \multicolumn{7}{|l|}{ Safety } \\
\hline Dropped needles & $3.6(3.5)$ & $5.8(6.8)$ & $10.8(10.4)$ & 0.002 & 0.039 & 0.268 \\
\hline Unnecessary piercing & $2.6(2.6)$ & $6.3(5.0)$ & $10.4(8.4)$ & $<0.001$ & 0.036 & 0.004 \\
\hline Needle outside of view & $3.3(4.8)$ & $6.2(9.9)$ & $8.1(11.2)$ & 0.136 & 0.534 & 0.301 \\
\hline Suture breakage & $0.0(0.0)$ & $0.3(0.5)$ & $0.6(1.2)$ & 0.007 & 0.133 & 0.016 \\
\hline \multicolumn{7}{|l|}{ Task specific } \\
\hline Total knots & $1.5(0.9)$ & $1.3(0.7)$ & $1.1(0.4)$ & 0.123 & 0.324 & 0.373 \\
\hline Surgeon knots & $0.6(1.0)$ & $0.6(0.8)$ & $0.8(0.6)$ & 0.473 & 0.281 & 0.985 \\
\hline Needle precision & $96(8)$ & $79(30)$ & $90(13)$ & 0.042 & 0.101 & 0.008 \\
\hline Total time & 171 (77) & 277 (172) & 546 (328) & $<0.001$ & $<0.001$ & 0.011 \\
\hline
\end{tabular}

Data in this table represents mean performance scores and standard deviation. $R$ Robotic experienced, $L$ Laparoscopic experienced, $N$ Novices. $P$-values were calculated using independent $t$-test, values of $<0.05$ were considered statistically significant (displayed in bold)

'total time'. Task 3 consists of the parameters; 'path length left', 'instruments collisions', 'precise needle passages' and 'total time'. The 'times out of view' parameters and 'distance scope and tissue' parameters were not included in the composite score because the novice group outperformed the robotic group on the 'times out of view' parameter.

The results for the contrasting group analysis using the composite scores are shown in Fig. 5. The cutoff values, theoretical false positive and false negative percentages were calculated between all three groups.
The lowest theoretical false positive/false negative percentage was found for Task 1 at a cutoff value of 73 and 74 between novice and laparoscopic participants versus robotic experienced $(21 / 5 \%$ and $31 / 6 \%)$. The mean composite score of Task 2 shows a gradual increase between the experience groups. The cutoff value between novice and laparoscopic participants was found at 85 and 88 with a false positive/false negative percentage of $28 / 4 \%$ and $45 / 11 \%$. The cutoff score for Task 3 shows the lowest discriminative ability between the novices and robotic experienced with $49 \%$ false positives and $22 \%$

Table 6 Mean (SD) performance outcomes per group on Task 3

\begin{tabular}{|c|c|c|c|c|c|c|}
\hline Task 3: Vaginal cuff closure & Robotic experience & Laparoscopic experience & Novices & $p$-values & & \\
\hline Movements & $n=15$ & $n=26$ & $n=29$ & R vs $N$ & L vs N & $R$ vs $L$ \\
\hline Path length left & $3813(1508)$ & $6862(3261)$ & $5544(2397)$ & 0.015 & 0.091 & $<0.001$ \\
\hline Path length right & $4784(1851)$ & $5941(2965)$ & $5377(2937)$ & 0.482 & 0.482 & 0.133 \\
\hline Movements left & $344(127)$ & $494(212)$ & $438(164)$ & 0.060 & 0.279 & 0.018 \\
\hline Movements right & $386(156)$ & $501(229)$ & $450(182)$ & 0.258 & 0.367 & 0.065 \\
\hline Entrance and exit points & $28(4)$ & $32(7)$ & $31(5)$ & 0.105 & 0.291 & 0.013 \\
\hline Distance scope and tissue & $94(20)$ & $116(10)$ & $120(1)$ & $<0.001$ & 0.056 & 0.001 \\
\hline \multicolumn{7}{|l|}{ Safety } \\
\hline Instrument collisions & $9(11)$ & $32(32)$ & $24(19)$ & 0.001 & 0.280 & 0.002 \\
\hline Times out of view & $32(34)$ & $16(11)$ & $10(16)$ & 0.030 & 0.148 & 0.088 \\
\hline Unnecessary piercing & $10.8(8.4)$ & $14.4(11.1)$ & $13.1(10.1)$ & 0.460 & 0.647 & 0.284 \\
\hline Suture breakage & $0.0(0.0)$ & $0.0(0.2)$ & $0.0(0.2)$ & 0.479 & 0.939 & 0.455 \\
\hline \multicolumn{7}{|l|}{ Task specific } \\
\hline Precise needle passages & $10.3(3.0)$ & $12.0(3.9)$ & $12.4(3.0)$ & 0.032 & 0.661 & 0.153 \\
\hline Needle passages & $14.1(1.8)$ & $16.1(3.3)$ & $15.3(2.5)$ & 0.105 & 0.291 & 0.013 \\
\hline Total time & $423(180)$ & $580(250)$ & $573(210)$ & 0.024 & 0.916 & 0.040 \\
\hline
\end{tabular}

Data in this table represents mean performance scores and standard deviation. $R$ Robotic experienced. $L$ Laparoscopic experienced. $N$ Novices. $P$-values were calculated using independent t-test. Values of $<0.05$ were considered statistically significant (displayed in bold) 


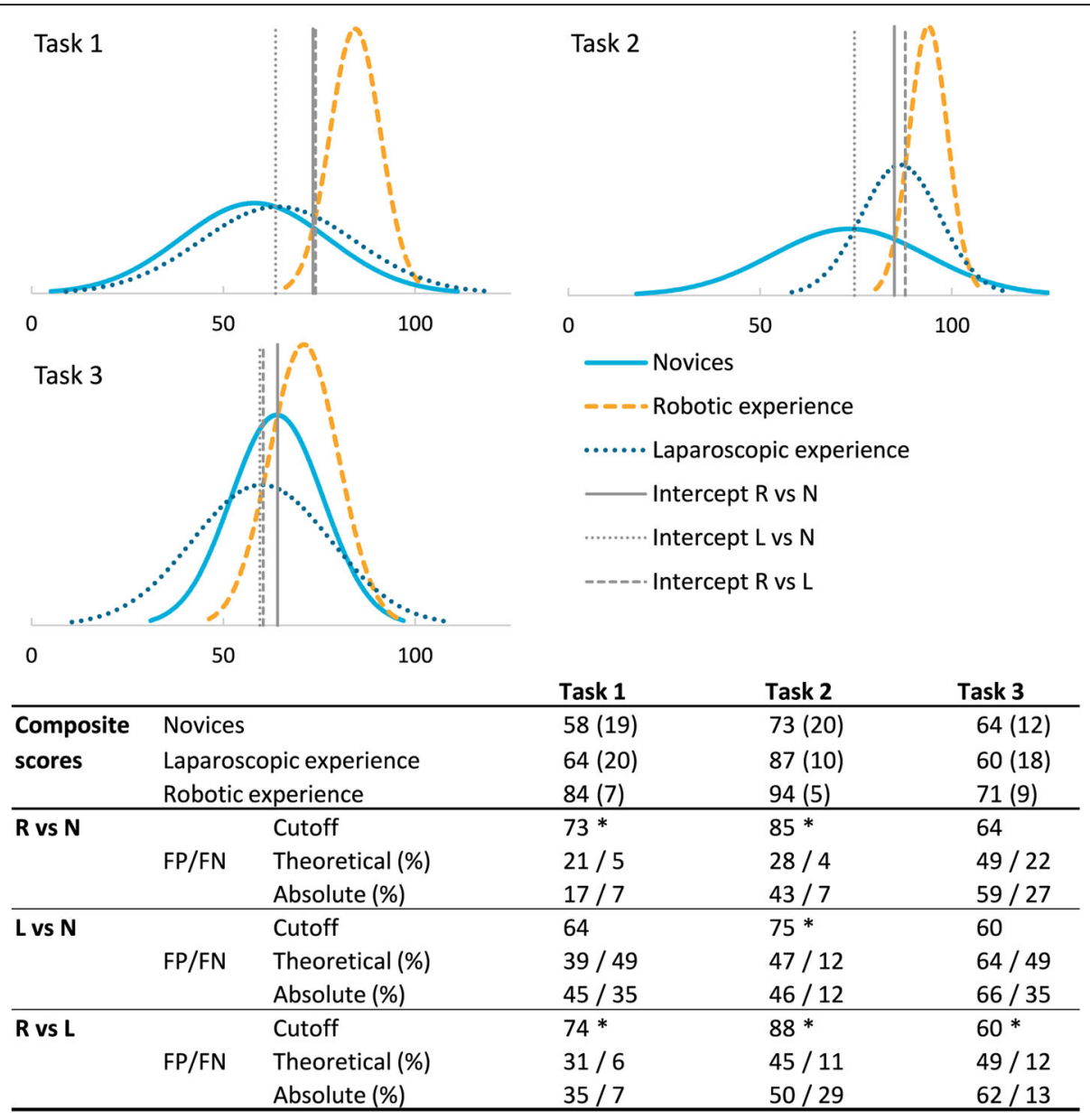

Fig. 5 Mean composite score outcome and contrasting group analysis results of Task 1-3 with the corresponding theoretical and absolute false positive (FP) and false negative (FN) percentages. Data in this table represents mean composite outcomes with standard deviation. Cutoff values were calculated using the contrasting groups method. $R=$ Robotic experienced. $L=$ Laparoscopic experienced. $N=$ Novices. $F P=$ false positive percentage, $\mathrm{FN}=$ false negative percentage. ${ }^{*}$ indicates a $p$-value $<0.05$ between the corresponding groups based on the mean composite score

false negatives. A sub analysis was performed for each task by weighing the included parameters in a best/worst case scenario, however, this did not result in a significantly better discriminative ability.

\section{Discussion}

In this study the levels of validity evidence were assessed according to Messick's framework [11, 16] for three suturing tasks on the RobotiX VR simulator. Results show a positive content validity evidence, with room for improvement regarding the realism of all three tasks. The usability was scored good to excellent particularly by the laparoscopic (target) group (means 4.2-4.3). Additionally, the didactic value was scored good by the robotic experienced participants for all three tasks (means 3.94.0). The relationship to other variables and the consequence evidence validity resulted in a usable composite score with an accompanying pass/fail score for the tilted plane needle transfer (Task 1) and intracorporal suturing
(Task 2) tasks. These scores allow for valid proficiencybased training which can be implemented in a robot assisted curriculum to assess the skills of a trainee. The third task (anastomosis needle transfer) seemed to be either too difficult for our expert group or was too strict in the assessment parameters to result in a valid composite score (Fig. 5). The laparoscopic experienced were unable to show adequate discriminative ability from the novices and robotic experienced group based on the composite score (Fig. 5). Although, the laparoscopic experienced were able to show some construct parameters and higher average composite score outcomes versus the novice group for Task 1 and 2 .

Previous validation studies were performed regarding the validity of the RobotiX simulator [22-28]. However, only limited studies were performed using the contemporary framework of validity [24]. The manuscript by Hovgaard et al. recently studied the Vaginal cuff closure task (Task 3 in this study) and found similar parameter 
outcomes as this study [24]. Construct between novices and robotic experienced participants was found in both studies for the 'path length', 'instrument collisions' and 'total time' parameters. Although our study also found construct for the 'precise needle passages' parameter, it was not shown for the 'unnecessary piercing points' parameter, as Hovgaard et al. found. Interestingly, they reported that robotic experienced participants used the camera functionality significantly more, therefore working closer on the target area and scoring significantly higher on the out of view parameters [24]. This effect was also shown in the current study, with a statistically significant difference in the 'distance scope and tissue' and 'times out of view' parameters between the novice and robotic experienced group. Consequently, this makes the 'out of view' parameter unfit for the proficiency composition if not corrected for the distance. However, when learning robot assisted surgery it is important in terms of safety to keep instruments in view at all time, due to the lack of haptic feedback. This may also indicate the potential pitfalls of using experienced robotic surgeons. The calculated pass/fail score by Hovgaard et al. was based on participants fifth and sixth repetition of a learning curve which showed an absolute false positive and false negative percentage of 36 and $27 \%$ respectively. This study shows a similar false negative percentage (27\%) but is unable to reproduce the false positive percentage ( $36 \%$ in this study). Possible differences are the parameters included for the composite score calculation, number of participants (11 novices and 11 robotic experienced versus 15 robotic experienced and 19 novices in this study) and the number of repetitions that participants performed.

The three main strengths of this study are the relatively high number of participants $(n=70)$, the inclusion of the laparoscopic participants as the target group to learn robot assisted surgery and the calculation of composite scores for multiple tasks. However, there are some limitations to this study as well. The novice group scored the usability significantly higher compared to robotic experienced for all tasks. Although, both groups were highly positive, this result shows a possible influence by the novelty of this technique for the novice group. Therefore, positive conclusions on base of the novice group are limited. The performance results showed a valid composite pass/fail score for Task 1 and 2 however, for Task 3 the composite pass/fail score resulted in a higher percentage of false positives and false negatives which indicates a poor sensitivity and specificity. This is most likely because the construct validity was only shown for four out of twenty-five parameters provided by the simulator. Interestingly, in this specific group, there were more statistically significant differences in the parameters of the laparoscopic versus the robotic experienced group than for the novice versus robotic group (six versus four parameters). This could be due to the inexperience of the novice group, which caused more careful handling and therefore, better performance. Concerning the intracorporal suturing (Task 2 ), the main goal was the correct knot placement, although this study could not show construct validity for any knot specific parameter. Also, during Task 2 multiple participants noticed errors with the simulated suture itself in this task, which led to the system scoring the tied knot as a single wrap where a double was placed. This limitation causes the calculated pass/fail score to be unable to score a trainee on the correctness of the knot. Results from all three tasks showed limited parameters with construct despite the wide variety of parameters available. This limitation in construct is also shown in previous studies $[24,28]$. Therefore, a subexpert analysis was performed (not shown) to assess increase of construct parameters using only more experienced robotic participants. However, this resulted in no additional parameters establishing construct validity.

Corresponding to the training of airline pilots, a training curriculum for robot assisted surgery should be composed of multiple modalities from which VR training is a single component [2]. Next should be the implementation of the tasks for proficiency-based training in a specific curriculum, in which the pass/fail limit should be reached before using other methods such as proctoring. Complemented by other training modalities, the proficiency-based VR training can be used to individually train component steps of specific procedures. These component steps should be validated in other simulation models to assess the transfer of skills.

\section{Conclusion}

This study shows evidence of validity on the response, content relation to other variables and consequence levels for three suturing tasks on the RobotiX robot assisted simulator. The calculated composite pass/fail scores can be used for proficiency-based training with adequate discriminative power between novice and robotic experience in the tilted plane needle transfer and intracorporal suturing tasks. This can be implemented for trainees with or without laparoscopic experience as a proficiency goal in a robot assisted surgery training curriculum, supporting optimal training before starting with patient related robot assisted surgery.

\section{Supplementary information}

Supplementary information accompanies this paper at https://doi.org/10 1186/s12893-020-00839-z.

Additional file 1. Questionnaire used in this study.

Additional file 2 . 


\section{Abbreviations}

VR: Virtual reality; FP: False positive; FN: False negative

\section{Acknowledgements}

We would like to thank 3D-systems for providing accessibility to their simulator.

\section{Authors' contributions}

EL contributed to the design, acquisition, analysis, interpretation and draft of the work. IB and CR contributed to the interpretation and revision of the work. SB contributed to the conception, design, analysis, interpretation and revision of the work. All authors read and approved the final manuscript.

\section{Funding}

No funding was provided for this study.

\section{Availability of data and materials}

The datasets used and analyzed during the current study are available as an additional supporting file.

\section{Ethics approval and consent to participate}

Ethical approval and consent for anonymous gathering of opinion and performance data was stated in Supplemental 1 which was read and signed when entering the study. Due to the voluntary non-medical setup without any invasive interventions of this study no ethical committee approval was required [29].

\section{Consent for publication}

Written consent for publication has been obtained from the person shown in Fig. 1.

\section{Competing interests}

$\mathrm{CR}$ is a member of the editorial board of BMC Surgery working as an Associate Editor and was therefore not involved in the publication process.

The authors EL, IB and SB declare that they have no competing interests.

Received: 20 February 2020 Accepted: 30 July 2020

Published online: 12 August 2020

\section{References}

1. Intuitive Surgical Incorporated. Intuitive Surgical Annual Report. 2018. http://www.annualreports.com/Company/intuitive-surgical-inc. Accessed 28 Oct 2019.

2. Collins JW, Wisz P. Training in robotic surgery, replicating the airline industry. How far have we come? World J Urol. 2019:38:1645-51.

3. Schreuder HW, Wolswijk R, Zweemer RP, Schijven MP, Verheijen RH. Training and learning robotic surgery, time for a more structured approach: a systematic review. Bjog. 2012;119(2):137-49.

4. Zorn KC, Gautam G, Shalhav AL, Clayman RV, Ahlering TE, Albala DM, et al. Training, credentialing, proctoring and medicolegal risks of robotic urological surgery: recommendations of the society of urologic robotic surgeons. J Urol. 2009;182(3):1126-32.

5. Hanly EJ, Miller BE, Kumar R, Hasser CJ, Coste-Maniere E, Talamini MA, et al. Mentoring console improves collaboration and teaching in surgical robotics. J Laparoendosc Adv Surg Tech A. 2006;16(5):445-51.

6. McDougall EM, Corica FA, Chou DS, Abdelshehid CS, Uribe CA, Stoliar G, et al. Short-term impact of a robot-assisted laparoscopic prostatectomy 'mini-residency' experience on postgraduate urologists' practice patterns. Int J Med Robot. 2006;2(1):70-4

7. Hart R, Karthigasu K. The benefits of virtual reality simulator training for laparoscopic surgery. Curr Opin Obstet Gynecol. 2007;19(4):297-302.

8. Alaker M, Wynn GR, Arulampalam T. Virtual reality training in laparoscopic surgery: A systematic review \& meta-analysis. Int J Surg (London, England) 2016:29:85-94.

9. Nagendran M, Gurusamy KS, Aggarwal R, Loizidou M, Davidson BR. Virtual reality training for surgical trainees in laparoscopic surgery. Cochrane Database Syst Rev. 2013;(8):Cd006575.

10. Larsen CR, Soerensen JL, Grantcharov TP, Dalsgaard T, Schouenborg L, Ottosen C, et al. Effect of virtual reality training on laparoscopic surgery: randomised controlled trial. Bmj. 2009;338:b1802.
11. Borgersen NJ, Naur TMH, Sorensen SMD, Bjerrum F, Konge L, Subhi Y, et al. Gathering validity evidence for surgical simulation: a systematic review. Ann Surg. 2018;267(6):1063-8

12. Schout BM, Hendrikx AJ, Scheele F, Bemelmans BL, Scherpbier AJ. Validation and implementation of surgical simulators: a critical review of present, past, and future. Surg Endosc. 2010;24(3):536-46.

13. Carter FJ, Schijven MP, Aggarwal R, Grantcharov T, Francis NK, Hanna GB, et al. Consensus guidelines for validation of virtual reality surgical simulators. Surg Endosc. 2005;19(12):1523-32.

14. Bric JD, Lumbard DC, Frelich MJ, Gould JC. Current state of virtual reality simulation in robotic surgery training: a review. Surg Endosc. 2016;30(6):2169-78.

15. Stefanidis D, Sevdalis N, Paige J, Zevin B, Aggarwal R, Grantcharov T, et al. Simulation in surgery: what's needed next? Ann Surg. 2015;261(5):846-53.

16. American Educational Research Association APA, National Council on Measurement in Education, Joint Committee on Standards for Educational and Psychological Testing. Standards for educational and psychological testing. Washington, DC: AERA; 2014.

17. Leijte E, Arts E, Witteman B, Jakimowicz J, De Blaauw I, Botden S. Construct, content and face validity of the eoSim laparoscopic simulator on advanced suturing tasks. Surg Endosc. 2019;33:3635-43.

18. Botden SM, Buzink SN, Schijven MP, Jakimowicz JJ. ProMIS augmented reality training of laparoscopic procedures face validity. Simul Healthc. 2008; 3(2):97-102

19. Botden SM, Berlage JT, Schijven MP, Jakimowicz JJ. Face validity study of the ProMIS augmented reality laparoscopic suturing simulator. Surg Technol Int. 2008;17:26-32

20. Likert R. A Technique for the measurement of attitudes. Arch Psychol. 1932; 140:5-55.

21. Jorgensen M, Konge L, Subhi Y. Contrasting groups' standard setting for consequences analysis in validity studies: reporting considerations. Adv Simul (London, England). 2018;3:5.

22. Whittaker G, Aydin A, Raveendran S, Dar F, Dasgupta P, Ahmed K. Validity assessment of a simulation module for robot-assisted thoracic lobectomy. Asian Cardiovasc Thorac Ann. 2019;27(1):23-9.

23. Watkinson W, Raison N, Abe T, Harrison P, Khan S, Van der Poel $H_{\text {, et al }}$ Establishing objective benchmarks in robotic virtual reality simulation at the level of a competent surgeon using the RobotiX Mentor simulator. Postgrad Med J. 2018;94(1111):270-7.

24. Hovgaard LH, Andersen SAW, Konge L, Dalsgaard T, Larsen CR. Validity evidence for procedural competency in virtual reality robotic simulation, establishing a credible pass/fail standard for the vaginal cuff closure procedure. Surg Endosc. 2018;32(10):4200-8.

25. Hertz AM, George El, Vaccaro CM, Brand TC. Head-to-head comparison of three virtual-reality robotic surgery simulators. JSLS. 2018:22(1):e2017.00081.

26. Harrison P, Raison N, Abe T, Watkinson W, Dar F, Challacombe B, et al. The validation of a novel robot-assisted radical prostatectomy virtual reality module. J Surg Educ. 2018;75(3):758-66.

27. Omar I, Dilley J, Pucher P, Pratt P, Ameen T, Vale J, et al. The RobotiX simulator: face and content validation using the fundamentals of robotic surgery (FRS) curriculum. J Urol. 2017:197(4):e700-e1.

28. Whittaker G, Aydin A, Raison N, Kum F, Challacombe B, Khan MS, et al. Validation of the RobotiX Mentor robotic surgery simulator. J Endourol. 2016:30(3):338-46

29. Radboudumc. Radboudumc Commission Human Related Research. 2019. https://www.radboudumc.nl/over-het-radboudumc/kwaliteit-en-veiligheid/ commissie-mensgebonden-onderzoek. Accessed 20 Feb 2020.

\section{Publisher's Note}

Springer Nature remains neutral with regard to jurisdictional claims in published maps and institutional affiliations. 